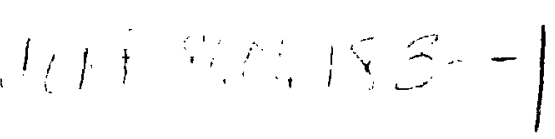

ON THE POSSIBLlity OF NEW PARTICLE PRODUCtion IN HEAVY-ION COLlislons*

(1)
A. B. Balantekin**

Physics Division

Oak Ridge National Laborator:

Oak Ridge, Tennessee 37831
CON $F-8606183--1$

DE86 014220

presented at

\author{
NATO International Advanced Course on \\ Physics of Strong Fields \\ Maratea, Italy \\ June $1-14,1986$
}

\title{
DISCLAIMER
}

This report was prepared as an account of work sponsored by an agency of the United States States Government nor any agency thereof, nor any of their Government. Neither the United States Government nor any agency legal liability or responsiemployees, rakes any warranty, express or implied, or assuinformation, apparatus, product, or bility for the accuracy, completeness, or usefulness of any inforinge privately owned rights. Referprocess disclosed, or represents that its use would nol inf or service by trade name, trademark, ence herein to any specific commercial product, process, or sem imply its endorsement, recommanufacturer, or otherwise does not necessarily constitute or any agency thereof. The views mendation, or favoring by the United States Government or any ate or reflect those of the

and opinions of authors expressed herein do not

\footnotetext{
* Research sponsored by the U.S. Department of Energy under contract DE-AC05-840R21400 with Martin Marietta Energy Systems, Inc.

**Present address: Department of Physics, University of Wisconsin-Madison, Madison, Hisconsin 53706
} 
ON THE POSSIBILITY OF NEW PARTICLE PRODUCTION IN HEAVY-ION COLLISIONS*

\author{
A. B. Balantekin ${ }^{\star \star}$ \\ Physizs Division \\ Oak Ridge National Laboratory \\ Oak Ridge, Tennessee 37831
}

\title{
INTRODUCTION
}

Experimental identification of narrow peaks in the positron spectra emtted in the low-energy collisions of heavy lons ${ }^{-4}$ has brought many sur-prises. Such structures were predicted to be present when the collision leads to a long-lived nuclear complex. ${ }^{5}$ However, the observed $\mathrm{z}$-dependence of the peak energy and the cross section does not seem to be consistent with the hypothesis that quasimolecular nuclear configurations are formed. The is binding energy for those systems is expected to vary strongly with the total nuclear charge $z .^{6}$ Hence, when this binding energy exceeds twice the electron miss, 7 the spontaileously created positron would have an energy strongly dependent on $z$. Inftial data ${ }^{1-3}$ indicated that there is at least one constant peak energy for all the systems investigated, supercritical or subcritical. More recent measurements ${ }^{8}, 9$ seem to unvell the existence of a multiple structure in the positron emission spectra, again with little Zdependence.

In this talk, I consider an alternative hypothesis, namely two-body decay of a particle, 10,11 which would explain the constancy of the peak energy. I also discuss the consequences of such a hypothesis and recent theoretical and experimental work motivated by $1 t$.

\section{PHENOMENOLOGY OF PARTICLE PRODUCTION}

I assume a pseudoscalar Interaction Lagrangian of the form ${ }^{10}$

$$
\mathscr{P}_{\text {int }}=g_{\mathrm{e}} \bar{\psi}_{\mathrm{e}} \mathrm{r}_{5} \psi_{\mathrm{e}} \phi_{\mathrm{a}}
$$

\footnotetext{
* Research sponsored by the U.S. Department of Energy under contract DE-AC05-840R21400 with Martin Marietta Energy Systems, Inc.

${ }^{\star *}$ Present address: Department of Physics, University of Wisconsin-Madison, Madison, Hisconsin 53706
} 
where the interaction s: ength $g_{e}$, and the mass of the particle, $m_{a}$, are free parameters to be fitted by the data. One can repeat similar analyses for other types of the interactions, $l !$ but a pseudoscalar interaction suffices to 1llustrate the basic principles. For the purposes of the analysis which follows, the Lagrangian in Eq. (1) can be elther elementary or effective. The mass of the particle is assumed to be greater than twice the electron mass, so that its dominant decay mechanism is 1nto electron-positron pairs. Since these positrons are products of a two-body decay, they would yield sharp peaks in the experimental spectrum. This hypothesis has a bullt-in assumption about the lifetime of the particle. It must live longer than heavy-ion reaction time $\left(\sim 10^{-20} \mathrm{sec}\right)$, but shorter than the time of flight to the positron detectors $\left(\sim 10^{-9} \mathrm{sec}\right)$. So if a subsequent analysis ylelds a lifetime outside the above limits, this hypothesis should be discarded.

The peak at $300 \mathrm{keV}$ positron kinetlc energy has three pleces of information: its energy, widh $(<70 \mathrm{keV})$, and the cross section $(\sim 200 \mu \mathrm{b}) .3^{3}$ The mass of the particle is adjusted to fit the peak energy, the production multiplicity to fit the integrated cross section, and the momentum to fit the width. One gets $10 \mathrm{~m}_{\mathrm{a}} \approx 1.6-1.8 \mathrm{MeV}$ momenta with less than $0.1 \mathrm{~m}_{\mathrm{e}} \mathrm{c}$ whth approximately $10^{-5}$ neutral particles being produced per reaction.

If we make a model for production, one can deduce the interaction strength ge from the multiplicity quoted above. Given the lack of definitive models, we can extract limfts on the interaction strength from the absence of this particle from other experimental observations. A particle interacting with electrons and positrons via the Lagrangian in Eq. (1) would contribute to various quantum electrodynamics processes. Here I constder the limits coming from the most thoroughly tested prediction of Q.E.D., the anomalous magneric moment of electron.10 A similar analysis has been sarried out for other Q.E.D. processes.12,13 For a particle with mass $1.6-1.8 \mathrm{MeV}$, the contribution to the anomalous magnetic moment is $\sim 5 \times 10^{-3} \mathrm{ge}_{\mathrm{e}}^{2} .10^{8}$ Thus, combining very acci ate recent measurements 14 and theoretical work on higher order Q.E.D. corrections, 15 one concludes that this contribution would not have been observed if ge $\sim 10^{-4} .10$ Since this particle predominantly decays into electron-positron pairs, its lifetime can then be calculated to be $\sim 10^{-13} \sec 10$ which is well within the previously mentioned bounds.

Light particles, even if they couple to matter very weakly, could carry away large amounts of energy from the stars and could have significant effects on other astrophysical processes. Now, using the constraints from red glant stars as an example, I illustrate that a particle, which would explain the heavy-ion data, would not contribute to the astrophysical processes. Light particles, if they directly couple to fermions as in Eq. (1), can be photoproduced in red giant stars and carry away energy. The observational bounds on energy loss is $100 \mathrm{ergs} / \mathrm{gr}-\mathrm{sec}$, and a significant portion of this could be due to the production of a particle if its mass is less than $5 \times$ $10^{2} \mathrm{keV} .15$ Obylously, this is much lighter than $1.8 \mathrm{MeV}$.

A cructal experiment to confirm the particle interpretation of heavyIon data is to measure the yield versus invariant mass by detecting electrons and positrons in colncidence. This is a very difficult experiment. However, it has been observed that the positrons assoctated with the narrow peak are correlated with the simultaneous emission of electrons whose energy spectrum also contains a narrow peak at approximately the same energy and with the same width. 8,17 For a detalled discussion of these experiments, I refer the reader to the other contributions to these Proceedings. 8,9 
The standard theory of strong interactions, quantum chromodynamlcs, does not necessarily have CP invariance, since gauge principle allows CP-violating terms. But there is, at present, no experimenial evidence for CP violation In strong interaction physics. Sometime ago, it was observed 8 that a global U(1) symmetry would suppress CP-violating 1nstanton effects. As a consequence of breaking thls $U(1)$ invarlance, one can 1ntroduce a neutral pseudoscalar particle, called an axion. 19 So far, all experiments try1ng to ooserve axlons have falled. One then might be tempted to rule out the particle Interpretation of heavy-ion data using the previous negative results from the axion search experiments. However, essentially all axion searches performed prior to 1986 are not sensitive to a particle decaying as fast as $10^{-13}$ sec. 10,20,21 A sumary of axion search expertments as of mid-1985 1s presented In Fig. 1 which displays the possible window.

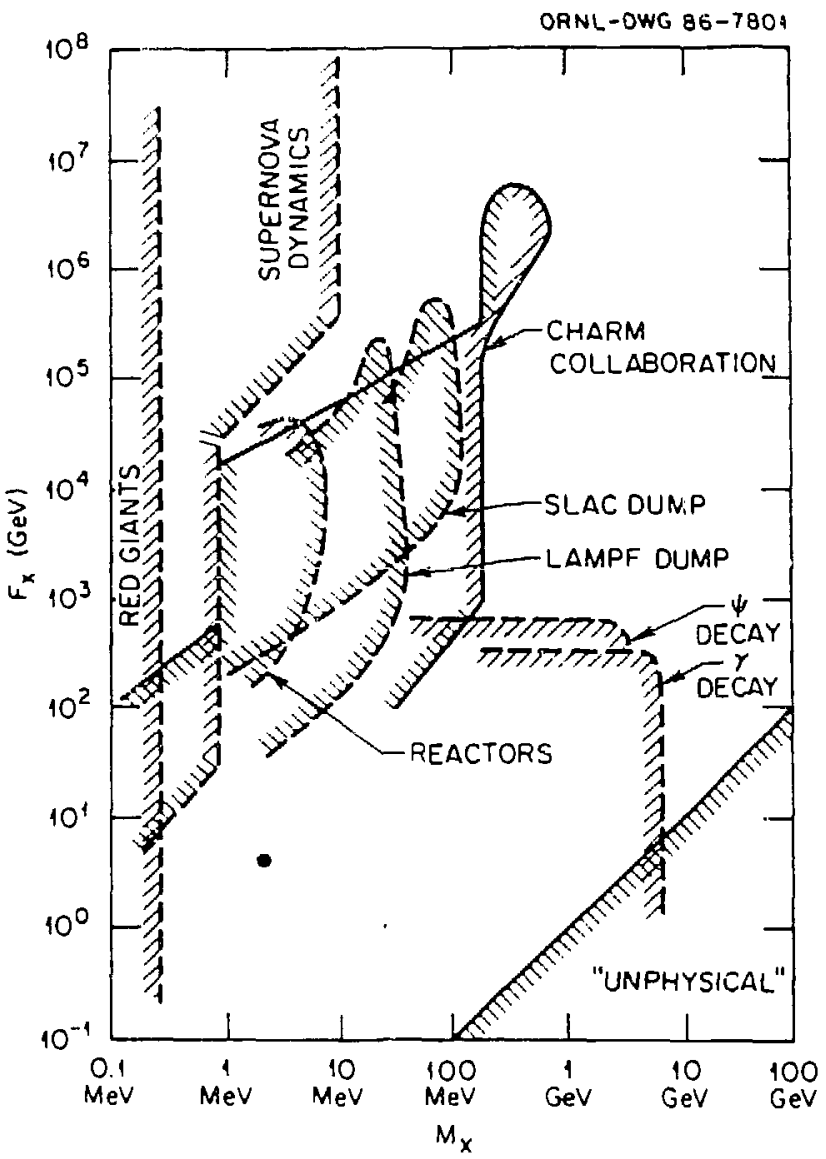

Fig. 1. A summary of world data for axion searches up to 1985 as presented by CHARM collaboration in $\operatorname{Re}$. 22. $F_{X}=250 \mathrm{X} \mathrm{GeV}$ and $\mathrm{M}_{\mathrm{x}}$ is the axion mass. The shaded areas in the parameter space are excluded by the specifled considerations. The CHARM collaboration data are from the $400-\mathrm{GeV}$ proton beam dump experiment reported 1n Ref. 22. The black dot denotes the parameters of the particle which would explain the heavy-ion data. 
In the standard axion modeli9 one Introduces two Higgs doublets. The ratio of the vacuum expectation values of these two fields is denoted by $x$. If the generic Lagrangian in Eq. (1) represents the coupling of axion to electron, the interaction strength $g_{e}$ can be related to $x$

$$
X=m_{e}\left(G_{F} \sqrt{2}\right)^{1 / 2} / g_{e}
$$

where $G_{F}$ is the Fermi constant. For an axion of mass 1.65 MeV, $x 1 s$ either $4.4 \times 10^{-2}$ or 22 assuming three generations of quarks and taking the ratin of $u$ and $d$ quark current algebra masses to be 0.56 . The latter $X$ value ylelds too long a lifetime $\left(\sim 10^{-6}\right.$ sec) to be compatible with experiments, lo while the former value gives a lifetime of about $10^{-12}$ sec, in agreement with the electron anomalous magnetic moment considerations.

As I enumerate in the next section, recent experiments and reanalyses of old data to accommodate the short axion lifetime still wles out the standard axion model. In particular, it was found out that if there $1 \mathrm{~s}$ a neutral, pseudoscalar boson of mass $1.6 \mathrm{MeV}$, 1t should couple to l1ght quarks more strongly than to heavy quarks. In the past year, a number of new models, taking this universality breaking into account, have been proposed. $23-26$ Again in the next section, I compare two of those models, those of Krauss and W1 $1 \mathrm{czek}^{23}$ and of Peccel et al..$^{4}$ with the recent experiments.

\section{RECENT PARTICLE SEARCH EXPERIMENTS}

Radiative Upsilon Decays

A search for possible radiative decays of heavy vector mesons into light pseudoscalar particles is experimentally feasible since the monochromatic photons provide a clear signal. Let us consider a heavy vector meson composed of the quark $q$ and 1 ts antlquark $\bar{q}$ and assume that this quark couples to the light particle vla a pseudoscalar interaction Lagrangian simllar to that in Eq. ( 1 ) with a coupling strength $\mathrm{gq}$. The ratio of the radiative decay width of the vector meson to the decay width into $\mu_{\mu}-$ pairs is given by 27

$$
\frac{\Gamma(V+\alpha+\gamma)}{\Gamma\left(V \rightarrow \mu^{+}+\mu^{-}\right)}=\frac{g_{q}^{2}}{2 \pi \alpha}\left(1-\frac{m_{a}^{2}}{m^{2}}\right)
$$

where $\mathrm{m}_{\mathrm{v}}$ is the mass of the vector meson and the quantum chromodynamics corrections are neglected.

$$
\text { For the decay of upsilon, Eq. (3) predicts }
$$

$$
B[Y: 1 s)+Y+a] \sim(2.7 \pm 0.7) \times 10^{-4} X^{2}
$$

In the standard axion model. 19 prevlous experfmental searches looking for this decay reported null results, 28 but they have assumed that the axion escapes the detector region before decaying. However, axions with a life-time of $\sim 10^{-13}$ sec would decay dominantly instde the detector. For $X=0.04$, Eq. (4) gives a branching vat1o of $0.16 \pm 0.04$. Two searches for axions decaying into $e^{+} e^{-}$inside the detector were recently performed by groups using the CUSB 29 and CLEO ${ }^{30}$ detectors. Both groups report an upper 11 mit of $\sim 2 \times 10^{-3}$ for the branching ratio which conclusively excludes the possibility of short-lived axions within the original model.

One can, nevertheless, use $\mathrm{Eq}$. (3) in conjunction with the above experImental results to obtain an upper limit on $g_{b} \cdot{ }^{21}$ Those limits would be 
consistent with the new axion models 23,24 since in these models the couplings of the axion to heavy quarks are suppressed.

Electron Beam Dump Experlments

A recent search for neutral penetrating particles has been performed using a 2.5-GeV electron beam at the National Laboratory for High Energy Physics (KEK) In Japan.31. After the beam hits a tungsten target, the axions were supposed to penetrate a dump and to decay into $e^{+} e^{-}$palrs in a decay volume. Neutral particles are assumed to be produced by the Primakoff process by photons

$$
\mathscr{Z}=\frac{\mathrm{g}_{\mathrm{Y}}}{\mathrm{m}_{\mathrm{e}}} \mathrm{F}_{\mu \nu} \widetilde{\mathrm{F}}^{\mu \nu} \phi_{\mathrm{a}}
$$

as wel1 as by bremstrahlung from electrons (cf. Eq. ( 1 )). A portion of the two-dimensional parameter space of $\alpha_{e}=g_{e}{ }^{2} / 4 \pi$ and $\alpha_{Y}=g_{\gamma}{ }^{2} / 4 \pi$ is excluded at the $90 \%$ confidence leve ${ }^{31}$ as suming $\mathrm{m}_{\mathrm{a}}=1.8 \mathrm{MeV}$. In particular, neutral particles living longer than $10^{-12} \mathrm{sec}$ are excluded for all values of ge, when $a_{Y} \geq 10^{-21}$. Also, when $g_{Y}=0$, the 1nterval $0.022<x<0.074$ is excluded for the standard axion model. Let me relterate that a standard axion would require $X \sim 0.04$ to expla1n the heavy-1on data.

ituclear Ml Transition Experiments

If there 1s enough energy, a neutral particle coupling to up and down quarks can be produred in nuclear transitions. 32 This is an ideal situation in which to use perturbation theory since the relevant couplings are small. Furthermore, since a neutral, pseudoscalar particle acts like a "magnetic" photon, 19 the emission of such a particle would follow the same selection rules as the magnetic transtitions in nucle1.33,34 Consequently, one could look for the decay of a nuclear state by neutral particle emission in competition with, say, Ml transitions.

The decay of the $9.17-\mathrm{MeV} \mathrm{J}^{\pi}=2^{+}, \mathrm{T}=1$ state $1 \mathrm{n}{ }^{14} \mathrm{~N}$ has been recently 1nvestigated for this purpose. 35 This state decays into the $J^{\pi}=1^{+}, T=0$ ground state by an Ml transition with a branch of $85 \%{ }^{36}$ Savage et al. measured the branching ratio of neutral particle emission decay to that of Ml transition by studying the angular correlation of $e^{+} e^{-}$patrs. 35 Assuming $\mathrm{m}_{\mathrm{a}}=1.7 \mathrm{MeV}$ and a 11 fetime less than $10^{-11} \mathrm{sec}$, they obtain an upper $11 \mathrm{mit}$ of $4 \times 10^{-4}$ for this branching rat1o, as opposed to the standard axion model prediction of 0.55 . Since this transition is isovector, the above result can also be used to provide an upper limit of $1.4 \times 10^{-2}$ to the neutral particle-nucleon isovector coupling constant.

Other Limits from Nuclear $\mathrm{e}^{+} \mathrm{e}^{-}$Pair Measurements

Calaprice and his collaborators reanalyzed 37 early Brookhaven data 38 determining multipolarities of varfous nuclear electromagnetic transitions. In particular, they studied the decays of the $3.56-\mathrm{MeV} \sigma^{+}$state in ${ }^{6} \mathrm{LI}$, and the $7.03-\mathrm{MeV} 2^{+}$stace $1 n{ }^{14} \mathrm{~N}$ by internal palr conversion to the ground states of these nucle1. They deduced the branching ratio of neutral particle emission decay to the $e^{+} e^{-}$palr emlssion decay to be less than 0.14 for ${ }^{6} \mathrm{LI}_{1}$ and less than 5 for ${ }^{14} \mathrm{~N}$. The standard axion, 19 Krauss-W11czek, 23 and Pecce1 et al. 24 models predict this ratio to be $7.2(300), 10(0)$, and $10(0)$ respectively for ${ }^{6} \mathrm{LI}\left({ }^{14} \mathrm{~N}\right)$. Thus, these two new axton models seem to be also ruled out. 
NEUTRAL PARTICLE PRODUCTION MECHANISMS IN HEAVY-ION COLLISIONS

During the past year, considerable theoretical activity has been devoted to the investigation of various possible mechanisms to produce light, neutral particles in heavy-ion collisions.10,39-42 Generally, one assumes that the heavy-ion system generates a current $j(\vec{x}, t)$ which is the source of the neutral particles. The resulting inceraction Lagrangian

$$
\mathscr{L}_{\text {int }}=\phi_{\alpha} \mathrm{J}(\overrightarrow{\mathrm{x}}, \mathrm{t})
$$

leads to an exactly solvable quantum fleld theory problem. ${ }^{43}$ The momentum distribution, $F(\vec{k})$, of the produced particles 1 given by

$$
F(\vec{k}) \sim e^{-\bar{n}}\left|j\left(\vec{k}, \omega_{k}\right)\right|^{2} / \omega_{k}
$$

where $u_{k}=\sqrt{k^{2}+m_{a}^{2}}, \bar{n}$ is the average number of the particles produced, and

$$
j\left(\vec{k}, u_{k}\right)=\int d^{4} \vec{x} j(\vec{x}, t) \exp \left[1\left(\vec{k} \cdot x \rightarrow w_{k} t\right)\right] \text {. }
$$
take 10

The function $j$ was modeled in several ways. One possibility is to

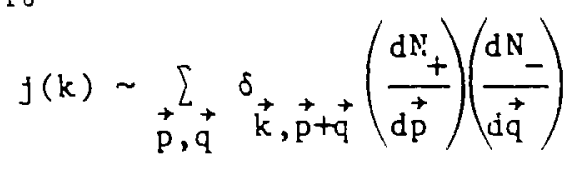

where $\left(d N_{4} / d \vec{p}\right)$ and $\left(d N_{-} / d \vec{q}\right)$ are the differential number of positrons and electrons respectively computed using the techniques of Ref. 44. Another possibility is to consider $r^{3-42}$ effective pseudoscalar

$$
f(\vec{x}, t) \sim \vec{E} \cdot \vec{B}
$$

or scalar

$$
f(\vec{x}, t) \sim \vec{E}^{2}-\vec{B}^{2}
$$

couplings to the classical electromagnetic field of heavy ions.

One can unfold the experimental data directly to obtain the momentum distribution given in Eq. (7). This had been done 1n Ref. 10, and the distribution shown in Fig. 2b (solid line) was obtalned. Probably the most salfent feature of this figure is that the momentum spread is very small $(\leq 0.1 \mathrm{~m} c)$. On the other hand, $F(k)$ obtalned by substituting Eq. (9) into Eq. (7) is much broader (Fig. 2b, clashed line). Other authors $39-42$ using the source terms given in Eq. (10), modeled along very different lines, arrive at a similar conclusion. Thus, the theory seems to fall in describing the production mechanism if indeed such particles are produced in heavy-ion collisions. However, at this conference it was reported that one can fold the broad distribution in Fig. 2 by the kinematical constraints of the experiment and obtain a rather sharp momentum spread.

\section{COMPOSITE AND EXOTIC PARTICLES}

So far, I have confined my discussion to the particles which are either elementary or wth some possible structure which cannot be probed at these energles. A mumber of other posstbilities were also considered concerning the creation of composite particles or exotic states which I w1ll summarize very briefly. 


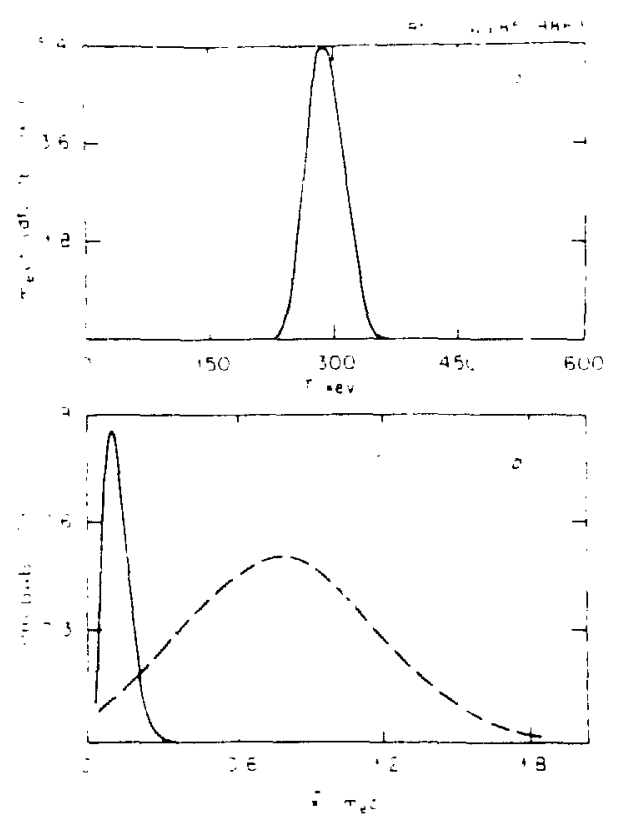

F1g. 2. a) Differential number of positrons emitted in the heavy-ion collisions. b) Momentum distribution of the neutral particles required to fit the data (solid line) and obtained from Eqs. (7) and (9) (dashed line).

Wong suggested that a polyelectron complex $\left(\mathrm{e}^{+} \mathrm{e}^{+} \mathrm{e}^{-}\right)$could be produced in heavy-ion col1isions. 45 The decay of this complex into a positron and a photon would create a sharp positron 11ne. However, the decay of such a complex is dominated by the two-photon mode $e^{+} e^{+} e^{-}+e^{+}+2 \gamma$. Indeed the branching ratio $\Gamma_{1 \gamma} / \Gamma_{2 \gamma}$ is calculated to be $\sim 10^{-1} 1.46$ Consequent $1 \mathrm{y}$, if the positron peak with a cross section of $200 \mathrm{\mu b}$ is due to the one-photon decay mode, most of the polyelectrons produced in heavy-ion collisions would decay through the two-photon mode ylelding a total positron cross section of $\sim 200$ $\times 10^{11} \mu b=2 \times 10^{7}$ barns. Such a situation is manifestly not realized in the experiments.

Celenza et al. suggested that these peaks could originate from the decay of nontopological solitons. 47 Other posstble mechanisms might be due to a change in the vacuum structure of a Higgs fleld 48 or due to the existence of systems with highly localized and tightly bound states of several electronpositron pairs. ${ }^{49}$ A detallea discussion of these subjects is glven elsewhere in this volume. 42,50

\section{CONCLUSIONS}

Experimental data still seem to allow a neutral particle with mass $\sim 1.6-1.8 \mathrm{MeV}$ and a lifetime of $\sim 10^{-12}-10^{-13} \mathrm{sec}$, but the situation might soon change. This particle is not the standard axion; the recent models proposed by Krauss-W1lczek and Peccel et al. also seem to be ruled out. Furthermore, if this particle is being produced in heavy-ion collisions, we do not know its production mechanism where the main difficulty appears to be obtalning the correct momentum spread. 
1. J. Schweppe et al., Phys. Rev. Lett. 51:2261 (1983).

2. M. Clemente et al., $\overline{\text { Phys. Let }}$. 1378:41 (1984).

3. T. Cowan et al., Phys. Rev. Lett. 54:1761 (1985).

4. H. Tsertos et al., Phys. Lett. 162B:372 (1985).

5. J. Rafelsk1, B. Müller, and W. Greiner, Z. Phys. A285:49 (1978).

6. W. Pieper and W. Greiner, Z. Phys. A218: $327 \overline{(1969) ; ~ S . ~ S . ~ G e r s h t e i n ~}$ and Y. B. Zeldovich, Lett. Nuovo C1m. 1:835 (1969); J. Rafelsk1, L. P. Fulcher, and W. Greiner, Phys. Rev. Lett. 27:958 (1971);

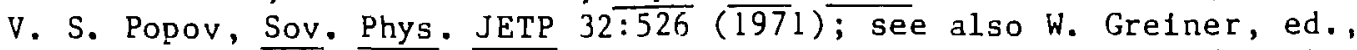
"Quartum Electrodynamics of Strong Flelds," Plenum, New York (1983).

7. B. Müller, W. Peltz, J. Rafelsk1, and W. Greiner, Phys. Rev. Lett. 28:1245 (1972).

8. T. Cowan, contribution to these proceedings.

9. H. Bokemeyer, contribution to these proceedings.

10. A. B. Balantekin, C. Bottcher, M. R. Strayer, and S. J. Lee, Phys. Rev. Lett. 55:461 (1985).

11. A. Schafer el al., J. Phys. G11:L69 (1985).

12. J. Reinhardt, A. Schafer, B. Müller, ard W. Grelner, Phys. Rev, C33:194 (1986).

13. J. Reinhardt, contribution to these proceedings.

14. R. S. Van Dyck, P. B. Schwluberg, and H. G. Dehmelt, Phys. Rev. Lett. $38: 310$ (1977).

15. T. Kinoshita and W. B. Lindquist, Phys. Rev. Lett. 47:1573 (1981).

16. A. Barroso and G. C. Branco, Phys. Tett. $116 \overline{B: 247}$ (1982).

17. T. Cowan et al., Phys. Rev. Lett. $5 \overline{6: 444}$ (1986).

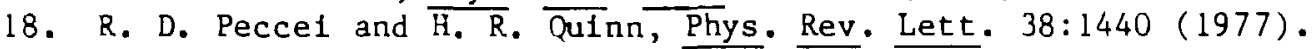

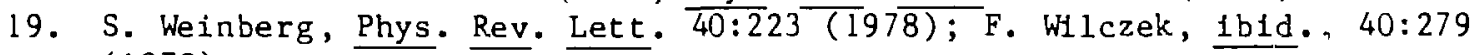
(1978).

20. N. C. Mukhopadhyay and A. Zehnder, Phys. Rev. Lett. 56:206 (1986).

21. A. B. Balantekin, C. Bottcher, M. R. Strayer, and S. J. Lee, in:

"Proceedings of Atomlc Theory Workshop on Relativistic and Q. E.D.

Effects in Heavy Atoms," H. P. Kelly and Y. -K. Kim, eds., American Institute of Physics, New York, p. 302 (1985).

22. F. Bergsma et al., Phys. Lett. 157B:458 (1975).

23. L. M. Krauss and F. Wliczek, Phys. Lett. 173B:189 (1986).

24. R. D. Pecce1, T. T. Wu, and T. Yanagida, Phys. Lett. 172B:435 (1986).

25. D. Y. Kim and M. S. Zahtr, U. of Regina preprint URTP-86-03 (1986).

26. E. Ma, Phys. Rev. D34:293 (1986).

27. F. Wilczek, Phys. Rev. Lett. 39:1305 (1977).

28. M. Sivertz et al., Thys. Rev. D26:717 (1982).

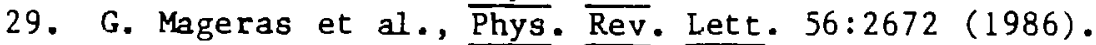

30. T. Bowcock et al., $\overline{\text { Phy } . ~} \overline{\text { Rev. }}$ Lett. $56: 2676$ (1986).

31. A. Konaka et al., Univers $\overline{1 t y}$ of Kyoto preprint (1986).

32. S. B. Treiman and F. Wilczek, Phys. Lett. 74B:381 (1978).

33. T. W. Donnelly et al., Phys. Rev. D18:1607 (1978).

34. A. Barroso and N. C. Mukhopadhyay, Phys. Rev. C24:2382 (1981).

35. M. J. Savage, R. D. Mckeown, B. W. Filippone, and L. W. Mitchell, Phys, Rev. Lett. 57:178 (1986).

36. F. Ajzenberg-Selove, Nucl. Phys. A360:1 (1981).

37. F. P. Calaprice, 1n: "Proceedings of Second Conference on the Intersections Between Particle and Nuclear Physics," D. F. Geesaman, ed., American Institute of Physics, New York (1986).

38. E. K. Warburton et al., Phys. Rev. 133B:42 (1964).

39. A. Chodos and L.C.R. Wifewardhana, Phys. Rev. Lett. 56:302 (1986);

D. Carzier, A. Chodos, and L.C.R. W1jewardhana, Yale preprint Y'P85-33 (1986).

40. K. Lane, Phys. Lett. 169B: 97 (1986).

41. B. Müller and J. Rafelski, U. of Cape Town preprint UCT-TP 40/1986.

42. B. Müller, contribution to these proceedings. 
43. C. Itzykson and J.-B. Zuber, "Quantum Field Theory," McGraw-Hill, New York, p. 163 (1980).

44. C. Bottcher and M. R. Strayer, Phys. Rev. Lett. 54:669 (1985).

45. C. Y. Wong, Phys. Rev. Lett. 56:1047 (1986).

46. M. C. Chu and V. Pönisch, Phys. Rev. C33:2222 (1986).

47. L. S. Celenza, V. I Mishra, C. M. Shakin, and K. F. Liu, Phys. Rev. Lett. 57:55 (1986).

48. A. Schäfer, B. Müller, and W. Greiner, Phys. Lett. 149B:455 (1984).

49. B. Müller, J. Reinhardt, W. Greiner, and A. Schäer, J. Phys. G12:L109 (1986).

50. A. 0. Barut, contribution to these proceedings. 